\title{
Prediction of forest NPP in Italy by the combination of ground and remote sensing data
}

\author{
Gherardo Chirici · Marta Chiesi - Piermaria Corona • \\ Nicola Puletti · Matteo Mura • Fabio Maselli
}

Received: 10 September 2014/Revised: 1 December 2014/Accepted: 12 January 2015

(C) Springer-Verlag Berlin Heidelberg 2015

\begin{abstract}
Our research group has recently proposed a strategy to simulate net forest carbon fluxes based on the coupling of a NDVI-driven parametric model, Modified C-Fix, and of a biogeochemical model, BIOME-BGC. The outputs of the two models are combined through the use of a proxy of ecosystem distance from equilibrium condition which accounts for the occurred disturbances. This modeling strategy is currently applied to all Italian forest areas using an available set of NDVI images and ancillary data descriptive of an 8-year period (1999-2006). The obtained estimates of forest net primary production (NPP) are first analyzed in order to assess the importance of the main model drivers on relevant spatial variability. This analysis indicates that growing stock is the most influential model driver, followed by forest type and meteorological variables. In particular, the positive influence of growing stock on NPP can be constrained by thermal and water limitations, which are most evident in the upper mountain and
\end{abstract}

Communicated by Arne Nothdurft.

G. Chirici ( $\triangle)$

geoLAB - Laboratory of Geomatics, Department of

Agricultural, Food and Forestry Systems, Università degli Studi

di Firenze, Via San Bonaventura, 13, 50145 Florence, Italy

e-mail: gherardo.chirici@unifi.it

M. Chiesi - F. Maselli

IBIMET-CNR, Via Madonna del Piano 10,

50019 Sesto Fiorentino, FI, Italy

P. Corona $\cdot$ N. Puletti

Consiglio per la ricerca in agricoltura e l'analisi dell'economia agraria, Forestry Research Centre (CRA-SEL), Arezzo, Italy

\section{Mura}

Dipartimento di Bioscienze e Territorio, Università degli Studi del Molise, Contrada Fonte Lappone snc, 86090 Pesche, IS, Italy most southern zones, respectively. Next, the NPP estimates, aggregated over seven main forest types and twenty administrative regions in Italy, are converted into current annual increment of standing volume (CAI) by specific coefficients. The accuracy of these CAI estimates is finally assessed by comparison with the ground data collected during a recent national forest inventory. The results obtained indicate that the modeling approach tends to overestimate the ground CAI for most forest types. In particular, the overestimation is notable for forest types which are mostly managed as coppice, while it is negligible for high forests. The possible origins of these phenomena are investigated by examining the main model drivers together with the results of previous studies and of older forest inventories. The implications of using different NPP estimation methods are finally discussed in view of assessing the forest carbon budget on a national basis.

Keywords Modified C-Fix · BIOME-BGC $\cdot$ Forest inventory - Current annual increment - Regional estimates · Italy

\section{Introduction}

The assessment of forest production is a central issue in applied ecology and is becoming increasingly important for evaluating the role of forest ecosystems as possible carbon sink (Hagedorn et al. 2001; Waring and Running 2007; Kolström et al. 2011). Information on forest production has been traditionally collected through user-driven national forest inventories (Corona and Marchetti 2007; Corona et al. 2011). In Italy, a national forest inventory (INFC) was recently completed (Gasparini et al. 2009a). One of the main objectives of this inventory is to provide an updated 